\title{
Alterstice
}

Revue internationale de la recherche interculturelle

International Journal of Intercultural Research

Revista International de la Investigacion Intercultural

\section{Regards pluriels sur les interventions sociales et de santé en contexte de diversité}

\section{Catherine Montgomery et Soumya Tamouro}

Volume 2, numéro 2, 2012

Regards pluriels sur les interventions sociales et de santé en contexte de diversité

URI : https://id.erudit.org/iderudit/1077560ar

DOI : https://doi.org/10.7202/1077560ar

Aller au sommaire du numéro

Éditeur(s)

Alterstice

ISSN

1923-919X (numérique)

Découvrir la revue

Citer ce document

Montgomery, C. \& Tamouro, S. (2012). Regards pluriels sur les interventions sociales et de santé en contexte de diversité. Alterstice, 2(2), 3-7.

https://doi.org/10.7202/1077560ar

\section{Résumé de l'article}

Ce numéro thématique met en lumière les travaux réalisés par les membres de l'équipe de recherche METISS (Migration et Ethnicité dans les Interventions en Santé et Service social). Au cours des quinze dernières années, l'équipe METISS est devenue une référence pour ses expertises relatives aux interventions sociales et de santé en contexte de diversité, notamment dans le contexte québécois. Les chercheurs de l'équipe METISS ont comme objectif de mieux comprendre les parcours migratoires et les différentes facettes de l'intégration des immigrants à leur société d'accueil. Le lien aux services sociaux et de santé est central à ces interrogations, mais les recherches entreprises s'intéressent aussi à d'autres dimensions pouvant contribuer à l'amélioration des conditions de vie des populations migrantes, comme le travail, l'interprétariat, l'insertion dans des réseaux de sociabilité ou la prise en compte des savoirs familiaux dans la relation d'aide. De façon générale, ces travaux nous invitent, d'une part, à poser un regard critique sur les conditions qui nuisent à la santé et aux conditions de vie des personnes migrantes et, d'autre part, à réfléchir à des pratiques d'intervention sociale et de santé plus inclusives. Cette façon d'appréhender les enjeux repose sur une conception dynamique de l'ethnicité et de l'immigration qui ne réduit pas le vécu des populations migrantes à des explications culturalistes, mais le situe plutôt dans la dynamique des parcours de vie des migrants et des rapports sociaux existant entre la société d'accueil et les nouveaux arrivants.
Ce document est protégé par la loi sur le droit d'auteur. L'utilisation des services d’Érudit (y compris la reproduction) est assujettie à sa politique d'utilisation que vous pouvez consulter en ligne.

https://apropos.erudit.org/fr/usagers/politique-dutilisation/ 


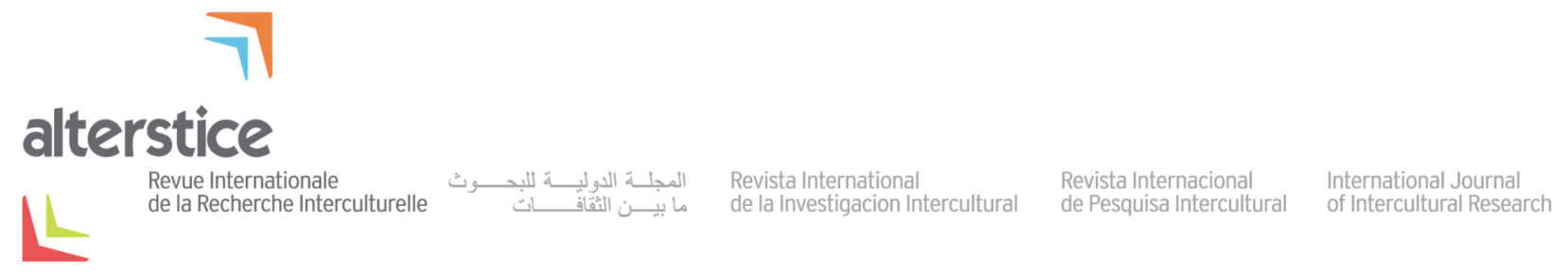

INTRODUCTION THÉMATIQUE

\title{
Regards pluriels sur les interventions sociales et de santé en contexte de diversité
}

\author{
Catherine Montgomery ${ }^{1}$ et Soumya Tamouro ${ }^{2}$
}

\section{Résumé}

Ce numéro thématique met en lumière les travaux réalisés par les membres de l'équipe de recherche METISS (Migration et Ethnicité dans les Interventions en Santé et Service social). Au cours des quinze dernières années, l'équipe METISS est devenue une référence pour ses expertises relatives aux interventions sociales et de santé en contexte de diversité, notamment dans le contexte québécois. Les chercheurs de l'équipe METISS ont comme objectif de mieux comprendre les parcours migratoires et les différentes facettes de l'intégration des immigrants à leur société d'accueil. Le lien aux services sociaux et de santé est central à ces interrogations, mais les recherches entreprises s'intéressent aussi à d'autres dimensions pouvant contribuer à l'amélioration des conditions de vie des populations migrantes, comme le travail, l'interprétariat, l'insertion dans des réseaux de sociabilité ou la prise en compte des savoirs familiaux dans la relation d'aide. De façon générale, ces travaux nous invitent, d'une part, à poser un regard critique sur les conditions qui nuisent à la santé et aux conditions de vie des personnes migrantes et, d'autre part, à réfléchir à des pratiques d'intervention sociale et de santé plus inclusives. Cette façon d'appréhender les enjeux repose sur une conception dynamique de l'ethnicité et de l'immigration qui ne réduit pas le vécu des populations migrantes à des explications culturalistes, mais le situe plutôt dans la dynamique des parcours de vie des migrants et des rapports sociaux existant entre la société d'accueil et les nouveaux arrivants.

\section{Rattachement des auteures}

${ }^{1}$ Département de communication sociale et publique, Université du Québec à Montréal, Montréal, Canada et équipe METISS (Migration et Ethnicité dans les Interventions en Santé et Service social); ${ }^{2}$ Alliance des communautés culturelles pour l'égalité dans la santé et les services sociaux (ACCESSS)

\section{Correspondance}

montgomery.catherine@uqam.ca

\section{Mots clés}

intervention interculturelle; services sociaux et de santé; altérité; clinique; diversité

\section{Pour citer cet article :}

Montgomery, C. et Tamouro, S. (2012). Regards pluriels sur les interventions sociales et de santé en contexte de diversité. Alterstice, 2(2), 3-8. 


\section{Introduction}

Ce numéro thématique met en lumière les travaux réalisés par les membres de l'équipe de recherche METISS (Migration et Ethnicité dans les Interventions en Santé et Service social) ${ }^{1}$. Au cours des quinze dernières années, l'équipe METISS est devenue une référence pour ses expertises relatives aux interventions sociales et de santé en contexte de diversité, notamment dans le contexte québécois. L'équipe bénéficie d'une structure de partenariat étroit entre les milieux universitaires et les milieux de pratique - principalement avec le Centre de santé et de services sociaux (CSSS) de la Montagne, un établissement sociosanitaire de soins de première ligne, mais aussi avec une vingtaine d'autres organismes publics et communautaires intéressés par les questions touchant à la santé et aux conditions de vie des personnes migrantes. Ce numéro d'Alterstice est représentatif de l'esprit de ce partenariat : il est issu d'une collaboration avec l'organisme ACCESSS (Alliance des communautés culturelles pour l'égalité dans la santé et les services sociaux), un regroupement d'organismes communautaires ayant comme objectif la représentation des intérêts des communautés ethnoculturelles auprès des instances décisionnelles en matière de santé et services sociaux.

Les travaux de METISS cherchent à mieux comprendre les parcours migratoires et les différentes facettes de l'intégration des immigrants à leur société d'accueil. Le lien aux services sociaux et de santé est central à ces interrogations, mais les recherches réalisées par l'équipe METISS touchent aussi à d'autres dimensions pouvant contribuer à l'amélioration des conditions de vie des populations migrantes, comme le travail, l'interprétariat, l'insertion dans des réseaux de sociabilité et la prise en compte des savoirs familiaux dans la relation d'aide. De façon générale, ces recherches nous invitent, d'une part, à poser un regard critique sur les conditions qui nuisent à la santé et aux conditions de vie des personnes migrantes et, d'autre part, à réfléchir à des pratiques d'intervention sociale et de santé plus inclusives. Cette façon d'appréhender les enjeux repose sur une conception dynamique de l'ethnicité et de l'immigration qui ne réduit pas le vécu des populations migrantes à des explications culturalistes, mais le situe plutôt dans la dynamique des parcours de vie des migrants et des rapports sociaux existant entre la société d'accueil et les nouveaux arrivants.

Dans cette perspective, la santé et le bien-être des personnes migrantes sont perçus comme relevant de l'interface entre différents acteurs - professionnels de la santé et des services sociaux, intervenants communautaires, réseaux de soutien locaux et transnationaux. Les articles présentés ici proposent des regards pluriels sur ces interfaces et sur leurs implications dans le soutien au processus d'établissement des nouveaux immigrants. Comment se construisent les pratiques d'intervention destinées à soutenir ce processus? Quelles sont les valeurs, implicites et explicites, qui façonnent les pratiques d'intervention? Comment les intervenants donnent-ils un sens à leur travail d'intervention? Quels sont les points de convergence, de négociation ou de compromis dans les rapports entre les différents acteurs, soit les institutions et organismes d'accueil, les intervenants et les personnes migrantes? Ces questionnements sous-tendent l'analyse des expériences d'intervention et de migration, tout en ouvrant un espace critique de réflexion pour repenser les façons d'intervenir et d'agir en contexte de diversité.

La santé et des conditions de vie décentes sont des valeurs fondamentales de la société québécoise. Les établissements publics de santé et de services sociaux ainsi que les organismes communautaires d'aide aux nouveaux immigrants sont de ce fait des milieux potentiellement significatifs pour l'intégration sociale des nouveaux immigrants ou de tout autre groupe minoritaire (OMS, 2010). Les établissements constituent en quelque sorte des agents de liaison entre les politiques étatiques en matière d'immigration et d'intégration d'une part et leur application concrète d'autre part (Verbunt, 2009). Pourvoyeurs de soins, de soutien psychosocial et de services de première nécessité, ce sont des acteurs de première ligne dans l'accompagnement des nouveaux arrivants dans le processus d'établissement et, de ce fait, constituent en même temps des lieux privilégiés de contact avec la société d'accueil (OMS, 2010). Cette capacité intégratrice est illustrée de différentes façons dans la littérature. Tandis que certains auteurs soulignent la créativité des savoirs d'intervention et la forte capacité des intervenants à

\footnotetext{
${ }^{1}$ L'équipe METISS bénéficie d'une subvention d'infrastructure du Fonds de recherche Québec - Société et Culture (FRQ-SC). L'équipe est gérée en partenariat entre le Centre de recherche et de formation du Centre de santé et de services sociaux (CSSS) de la Montagne et le département de communication sociale et publique de I'Université du Québec à Montréal (UQÀM). Nous tenons à remercier Andréanne Boisjoli, agente de valorisation des connaissances de l'équipe METISS, pour la coordination de ce numéro spécial.
} 
ajuster leurs façons de faire aux situations et contraintes rencontrées dans l'intervention en contexte de diversité, d'autres mettent de l'avant leur engagement social et l'esprit d'initiative qu'illustre le développement de projets porteurs permettant de mieux répondre aux besoins spécifiques d'une clientèle diversifiée (Cloutier, 2011; Racine, 2000; Saillant, Châteauneuf, Cognet et Charland, 2009). Sur tous ces plans, les établissements et organismes encouragent de nouvelles formes de vivre-ensemble, fondées sur des rapports d'entraide et d'accompagnement et sur la promotion de solidarités dans et à travers l'intervention.

Malgré ces forces, il demeure que l'ouverture des établissements et organismes à la diversité présente aussi des défis notables. Ces défis sont souvent formulés en termes d'enjeux d'adaptation et d'accessibilité aux services, observés la plupart du temps par le prisme des barrières de communication ou des conceptions différenciées de la santé et des soins (Bowen, 2001; Santé Canada, 2010). Du côté organisationnel, on note par exemple que la rencontre clinique peut durer plus longtemps (jusqu'à 2,9\% de plus) avec une clientèle nouvellement immigrante, mais que cette différence n'est pas toujours prise en compte dans la gestion des soins et services, dans la reconnaissance du temps supplémentaire nécessaire pour l'intervention ou dans la promotion d'outils permettant aux intervenants de mieux adapter leurs pratiques à la diversité (Battaglini, 2008 et 2010). Contraintes budgétaires et réorganisation des programmes et services, tant dans les secteurs publics que communautaires, constituent un autre frein important au développement de pratiques adaptées visant des clientèles spécifiques. D’autres travaux portent un regard plus critique sur l'intervention en contexte de diversité et nous amènent à réfléchir à la façon dont l'organisation des soins et services peut aussi participer à la construction de rapports d'exclusion des populations migrantes (Cloos, 2010; Cognet et Adam-Vezina, 2010; Cognet et Montgomery, 2007). De ce point de vue, les établissements et organismes d'aide n'interviennent pas dans un environnement neutre, mais véhiculent des valeurs et des façons de penser l'intégration des populations. Ces valeurs recèlent un potentiel de préjugés et de stéréotypes pouvant renforcer des représentations de l'altérité. Ainsi, la rencontre clinique elle-même devient le lieu où se négocient appartenances et valeurs différenciées. Sans être nécessairement préjudiciables, ces négociations identitaires caractérisent et orientent la relation d'aide en reproduisant des représentations du rapport entre « Nous » et l'« Autre » (Kanouté, Hohl, Xenocostas et Duong, 2007; Legault et Rachédi, 2008).

Les articles de ce numéro abordent les thèmes de solidarité, de négociation identitaire et d'altérité sous l'angle des pratiques et des savoirs d'intervenants et celui des expériences et savoirs des personnes migrantes. Ils proposent une lecture à la fois plus complexe et plus réelle des enjeux liés à la santé et au bien-être des personnes migrantes.

Le premier texte, de Marguerite Cognet et Sandra Bascougnano, nous rappelle que les pratiques de soins n'existent pas de façon isolée, mais s'insèrent plutôt dans un contexte historique et politique. Ainsi, la conception que se font les intervenants des pratiques et des relations de soins en contexte de diversité est nécessairement imprégnée des cadres sociopolitiques d'intégration et d'immigration qui prédominent dans une société donnée. Plus spécifiquement, les auteures proposent une analyse comparative des représentations des professionnels de la santé par rapport aux populations migrantes dans les contextes français et québécois, mettant en relief les figures d'altérité qui émergent dans la relation de soins. Bien que ces figures d'altérité soient tirées de situations contemporaines, on y observe des traces du rapport historique entre le colonialisme et la médecine qui marquent encore les modèles sociopolitiques d'intégration. En France et les départements outre-mer, celles-ci se manifestent dans la réactualisation de tensions historiques qui émergent dans la relation entre intervenant et client, le traitement différentiel de certains groupes et les rapports paternalistes qui s'introduisent dans la relation de soins. $\mathrm{Si}$, au Québec, la représentation des professionnels envers les migrants est généralement plus positive, les auteures observent néanmoins la persistance de stéréotypes pouvant renforcer l'asymétrie dans la relation de soins.

L'article de Josiane Le Gall, Spyridoula Xenocostas et Sophie Lemoyne-Dessaint aborde une autre dimension de la relation de soins, soit la place occupée par la spiritualité et la religion, sujets largement occultés au Québec depuis la Révolution tranquille, mais fortement médiatisés au cours des dernières années. Ces événements médiatiques ont projeté l'image d'un système de santé aux prises avec un sérieux problème de gestion de la diversité religieuse. Les auteures remettent en question cette image. En dépit de la multiplication de nouveaux groupes religieux au Québec au cours des dernières décennies, l'impact du pluralisme religieux sur l'organisation et la prestation des services de santé est encore largement peu documenté dans le contexte québécois. Les données recueillies lors de leur recherche sur les défis et enjeux posés par la diversité religieuse dans l'intervention de première ligne, au sein 
de CSSS montréalais, révèlent, d'un côté, que le processus de prise en compte des différences religieuses est défini en grande partie par les structures de santé elles-mêmes et, de l'autre côté, que la question religieuse, en plus d'être souvent confondue avec culture, est très peu abordée par les intervenants et qu'elle suscite un certain malaise chez plusieurs d'entre eux.

La rencontre clinique est aussi l'objet d'analyse dans I'article proposé par Isabelle Boivin, Yvan Leanza et Ellen Rosenberg portant sur le rôle des interprètes professionnels et familiaux dans la relation entre médecins et patients migrants. Dans un contexte de diversité, l'interprète est un acteur clé dont le rôle contribue à assurer l'accessibilité linguistique aux services, selon la Loi sur la santé et les services sociaux au Québec. Selon les auteurs, il est réducteur de limiter la compréhension du rôle de l'interprète à un simple acte linguistique. S'inspirant des travaux de Habermas, ils proposent une typologie des différents rôles joués par l'interprète dans la médiation entre le système médical (la voix du médecin) et le monde vécu du patient (la voix du patient). Les deux premières postures - celles de l'agent linguistique dont le rôle est de servir principalement de conduit d'information entre les médecins et les patients et celle de l'agent du système qui défend le discours institutionnel -- tendent à renforcer le discours médical et confirmer la prédominance de la voix médicale sur celle du monde vécu du patient. Quant aux deux autres postures - l'agent du monde vécu qui prend la défense des besoins et droits du patient et l'agent d'intégration qui accompagne le migrant dans différentes sphères d'activité liées à l'intégration - elles défendent avant tout la voix du patient. Ces postures sont différenciées selon le statut de l'interprète (professionnel ou familial), mais dans tous les cas les auteurs pensent que l'interprétariat devrait favoriser l'agir communicationnel, soit la reconnaissance de l'Autre dans la rencontre clinique.

L'article de Margareth Santos Zanchetta et collab. aborde la question de l'accessibilité linguistique aux services d'un autre point de vue, soit de la perspective des personnes migrantes. L'article s'interroge sur le cas spécifique des minorités francophones au Canada. Comme le démontrent les résultats, l'accessibilité aux services en français est plutôt restreinte ou même inexistante pour beaucoup de familles issues des minorités francophones hors Québec, ce qui les vulnérabilise de différentes façons. Sur le plan médical, la non-compréhension de la langue contribue à un faible niveau de littératie en santé de façon générale et, plus spécifiquement, à l'augmentation de l'anxiété visà-vis des diagnostics et des traitements, à l'impossibilité de juger de la qualité et de la pertinence des différentes sources d'informations disponibles, à l'augmentation du risque associé à la prise de décisions concernant leur santé et au manque de motivation envers des mesures préventives en santé. La non-compréhension de la langue a également des conséquences sur le plan social, notamment par le développement d'un sentiment d'isolement linguistique, particulièrement pour les femmes, et, aussi, d'un sentiment d'injustice lié au non-respect de leurs droits en tant que minorité linguistique. Ces conséquences renforcent leur statut minoritaire et les maintiennent dans une posture d'altérité, limitant par le fait même le respect du droit aux services dans les deux langues officielles du Canada, pourtant garanti dans les lois régissant les services sociaux et de santé.

Le respect du droit à la santé et le bien-être des personnes migrantes est également exploré dans l'article de Sylvie Gravel, Gabrielle Legendre et Jacques Rhéaume portant sur la participation des travailleurs migrants aux mesures préventives de santé et sécurité au travail (SST) dans des petites entreprises. D’un côté, les résultats suggèrent que les travailleurs sont peu au fait des mesures préventives de santé et sécurité au travail. Selon les personnes interrogées, les travailleurs migrants reçoivent moins de formation à l'embauche que les travailleurs d'origine canadienne, participent peu aux instances responsables de la SST et sont plus réticents à signaler des blessures ou utiliser des équipements de protection. De plus, les rapports d'autorité dans les entreprises font en sorte que les travailleurs migrants sont souvent mal placés pour signaler leurs préoccupations ou des risques potentiels. Du côté des employeurs, les résultats démontrent que les petites entreprises sont mal outillées pour faire la promotion des mesures de santé et sécurité au travail. Ceci est encore plus vrai parmi les employeurs et les superviseurs issus euxmêmes de l'immigration, qui de façon générale connaissent mal les mesures de SST. Face à ces constats, les auteurs insistent sur l'importance de favoriser une culture de SST dans les petites entreprises par des mesures ciblées afin que les droits et les obligations soient connus et respectés par tous.

Pour terminer la partie thématique de ce numéro, le texte de Nadia Deville-Stoetzel, Catherine Montgomery et Lilyane Rachédi aborde la question des conditions et des expériences de vie des personnes migrantes à partir d'une analyse des réseaux de soutien. Ces réseaux, informels pour la plupart, agissent en complémentarité du réseau 
institutionnel en santé et services sociaux. Ils participent tout particulièrement au renforcement des solidarités et, de ce fait, constituent des espaces positifs d'insertion. La migration internationale représente une mise à l'épreuve et une remise en question de toutes les sphères de la vie sociale : relations au quotidien, insertion résidentielle et professionnelle. Le rôle des réseaux a été exploré dans trois phases d'établissement de familles maghrébines au Québec, soit le projet migratoire enclenché dans le pays d'origine, la phase initiale de migration qui représente le moment de l'arrivée dans le pays d'accueil et la phase de l'établissement, qui correspond aux premières années suivant l'arrivée. Au cours de ces trois phases, à différents degrés, les réseaux familiaux, de compatriotes, de personnes provenant de pays d'accueil et d'organismes communautaires jouent un rôle important, que ce soit dans l'insertion résidentielle et professionnelle ou tout simplement dans la vie quotidienne.

Ainsi, l'intervention sociale et de santé en contexte de diversité est marquée par une dualité des rapports. Tandis que les uns sont fondés sur l'entraide, l'accompagnement et les solidarités, les autres reposent sur la négociation identitaire et l'altérité. Ces deux formes de rapports se côtoient dans l'organisation des services comme dans la relation d'aide, et constituent la trame des articles composant ce numéro spécial.

\section{Références bibliographiques}

Battaglini, A. (2008). "Exclusion et santé. Le rôle de la santé publique vis-à-vis des populations immigrantes ». Dans E. Gagnon, Y. Pelchat et R. Édouard (dir.), Politiques d'intégration, rapports d'exclusion. Action publique et justice sociale (p. 187-199). Québec: Presses de l'Université Laval.

Battaglini, A. (dir.) (2010). Les services sociaux et de santé et contexte pluriethnique. Montréal : Saint-Martin.

Bowen, S. (2001). Language barriers in access to health care. Ottawa : Santé Canada.

Cloos, P. (2010). « La racialisation, entre pouvoirs et savoirs : une lecture postcoloniale de la santé publique comme pratique discursive ", Aporia, 2, 41-48.

Cloutier, G. (2011). La valorisation des savoirs de femmes immigrantes en milieu communautaire. Source d'inspiration pour l'intervention sociale. Montréal : Éditions Vézina.

Cognet, M. et Adam-Vezina, E. (2010). L'observance : Analyse critique des savoirs (Rapport de recherche). Paris : Ministère de la Santé.

Cognet, M. et Montgomery, C. (dir.). (2007). Éthique de l'altérité. La question de la culture dans le champ de la santé et des services sociaux. Québec : Presses de l'Université Laval.

Kanouté, F., Hohl, J., Xenocostas, S. et Duong, L. (2007). « Les mots pour le dire et pour intervenir ». Dans M. Cognet et C. Montgomery (dir.), Éthique de l'altérité. La question de la culture dans le champ de la santé et des services sociaux (p. 241-260). Québec : Presses de l'Université Laval.

Legault, G. et Rachédi, L. (dir.). (2008). L'intervention interculturelle. Montréal : Gaëtan Morin.

Organisation mondiale de la santé (OMS). (2010). How health systems can address health inequities linked to migration and ethnicity. Copenhague : Organisation mondiale de la santé.

Racine, G. (2000). La production de savoirs d'action chez des intervenants sociaux. Paris : L'Harmattan.

Saillant, F. , Châteauneuf, D., Cognet, M. et Charland, M. (2009). " L'intervention auprès des réfugiés. Accueil, proximité, transformation ». Dans M. Clément, L. Gélineau et A.-M. McKay (dir.), Proximités. Lien, accompagnement et soin (p. 41-64). Québec : Presses de l'Université du Québec.

Santé Canada. (2010). La santé des migrants : Vers une approche axée sur les déterminants de la santé. Bulletin de recherche sur les politiques de santé, 17, 1-52. Ottawa : Santé Canada. http://www.hc-sc.gc.ca/srsr/pubs/hpr-rpms/bull/2010-health-sante-migr/index-fra.php\#a1

Verbunt, G. (2009). « L'intégration, un objectif du travail social? ». Dans G. Verbunt (dir.), La question interculturelle dans le travail social. Repères et perspectives (p. 15-46). Paris : La Découverte. 